\title{
Visual recognition under conditions of high and low discrimination redundancy
}

\author{
JAMES BOSCO, ROBERT M. W. TRAVERS, AND THOMAS WILKINS \\ WESTERN MICHIGAN UNIVERSITY
}

The purpose of this investigation was to measure the minimum interval for recognition under conditions of high and low discrimination redundancy. The minimum interval for recognition is the briefest interval between two stimuli at which the first stimulus can be processed. Two sets of four digits were used. Digits were selected so that one set exhibited high discrimination redundancy and the other low discrimination redundancy. Ten $S_{s}$ viewed digits with high discrimination redundancy, and $10 \mathrm{Ss}$ viewed digits with low discrimination redundancy. The minimum interval for recognition for Ss tested with high discrimination redundancy was nearly twice as large as the minimum interval for recognition of the digits with low discrimination redundancy.

Research has well established that the perception of a visual stimulus may be interfered with or masked by the presentation of a second visual stimulus (Sperling, 1960; Averbach \& Coriell, 1961). In the case of this particular masking phenomenon, the second stimulus appears to erase the information held briefly in a visual short-term storage system. The phenomenon is also widely referred to as the destructive erasure of an icon.

The minimum time that must be allowed between the initiation of the first stimulus and the appearance of the erasing stimulus in order for recognition of the first stimulus to take place is referred to here as the minimum interval for recognition (MIR). It represents the time required to process the incoming batch of information to the point where the recognition can take place. In a previous paper, techniques were described which can be used to measure the MIR (Travers \& Bosco, 1967). Various investigations have identified variables which affect the MIR. Luminance (Scharf, Zamansky, \& Brightbill, 1966), type of masking pattern (Schiller \& Wiener, 1963), and retinal locus (Forgus, 1966) have been identified as significant variables.

Another potentially salient variable is the discrimination redundancy of the set of test stimuli. In both the case of discrimination redundancy and its counterpart schematic redundancy, there is an excess of information. Yet, that which constitutes an excess of information when the task is memorization is not equivalent with an excess of information when the task is discrimination. If we consider any set of stimuli as composed of a schema set and a correction set, increasing the schema set (common elements) increases the schematic redundancy of the set, while increasing the correction set (distinctive elements) increases the discrimination redundancy of the set (Evans, 1967).

This study is concerned with the relationship between the discrimination redundancy of a set of stimuli and the minimum interval for recognition (MIR). In this investigation, two sets of four 2-digit numbers were used as test stimuli. In one set, no digit was used more than once (high discrimination redundant set). In the other set, each digit was used four times (low redundant set). The purpose of this investigation was to measure MIR under the conditions of high and low discrimination redundancy.

\section{PROCEDURE}

The test stimuli for this experiment were presented to the Ss through the use of a Gerbrands 2-field tachistoscope Model T-2B-1. Each stimulus consisting of a white digit on a black background was presented for $10 \mathrm{msec}$ followed by a dark field which varied in duration from $60 \mathrm{msec}$ to $6 \mathrm{msec}$. The dark field was followed by a blocking stimulus consisting of a complex white pattern on a black background and was presented for $10 \mathrm{msec}$. The test and blocking stimulus appeared between two dim red markers $2 \mathrm{~mm}$ in diameter. Subjects were instructed to respond after each trial. If they did not feel certain of the identification of the test stimulus, they were instructed to guess.

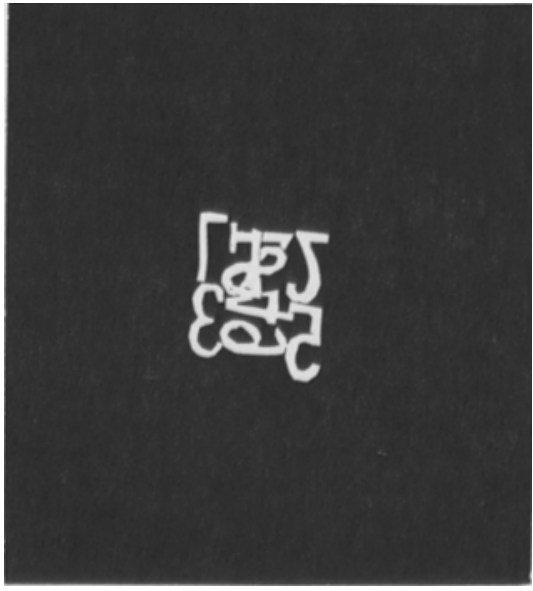

Fig. 1. Masking stimulus.

\section{DESIGN}

The digits used in the experiment were of two types: high discrimination redundancy and low discrimination redundancy. Table 1 shows the test stimuli.

Inspection of this table reveals that, in the case of high discrimination redundancy set of digits, if either of the two characters is identified, and if the set is known, it is possible to identify the stimulus. For the low discrimination redundancy set of stimuli, both characters must be observed to correctly identify the stimulus. The words and digits were presented in white print (Para-Tipe 36 point Gothic) with a luminance of $3.2 \mathrm{ft}-\mathrm{L}$ measured with a Spectra Brightness Spot Meter, Model UB. The letters were $3 / 8 \mathrm{in}$. high and $23 \mathrm{in}$. distant which results in a visual angle of less than $1 \mathrm{deg}$.

Each $S$ was tested on either the high or low redundant digits. The digits were presented in 10 blocks of eight in random order within each block with each of the four digits appearing twice in each block. Each of the first eight stimuli were presented followed by a dark field of $60 \mathrm{msec}$ followed by the blocking stimulus. Subsequent groups of stimuli had inter-exposure dark fields of 54, $48,42,36,30,24,18,12$, and 06 msec.

The general nature of the experiment was described to each $\mathrm{S}$, who was also shown a card with the pairs of digits to be used printed on it. Each $\mathbf{S}$ was given five trials with each of the pairs of digits alone followed by an additional five trials with the blocking stimulus presented after an interval of $200 \mathrm{msec}$. In addition, each $S$ was given three trials with the blocking stimulus immediately following the letter in order to familiarize him with the erasure effect and to explain that he was expected to guess a letter if not certain of the letter presented. The sequence dark field-digitdark field-masking stimulus-dark field was triggered by $S$ pressing a switch. Between trials $\mathrm{S}$ was asked to look away from the apparatus into the dimly lit experimental room. The entire procedure occupied about $20 \mathrm{~min}$.

\section{Table 1}

Test Stimuli

High Discrimination Redundancy Low Discrimination Redundancy

$\begin{array}{ll}15 & 26 \\ 26 & 22 \\ 37 & 62 \\ 48 & 66\end{array}$




\section{SUBJECTS}

These were 20 undergraduate students from Western Michigan University who volunteered for the experiment. The Ss were given credit in an introductory psychology course for participation by obtaining credit points.

\section{RESULTS AND DISCUSSION}

MIR for each of the 20 protocols was identified by selecting the point on the scale immediately below the S's last three consecutive correct responses. If there were three consecutive incorrect responses above this point, however, then the procedure of finding the three consecutive right responses above this point was again utilized.

Inspection of the data revealed that the MIR for Ss tested with high redundant digits was nearly twice as large as the MIR for Ss tested with low redundant digits. The means for the two groups were: high redundant $25.8 \mathrm{msec}$; low redundant $47.4 \mathrm{msec}$. The variance of the two groups was not significantly different at the .05 level. The value for $t$ was computed to be $18.02(p<.0005)$.

The data from this experiment strongly support the conclusion that discrimination redundancy facilitates the recognition of digits. Previous investigations (Long, Reid, \& Henneman, 1960; Reid, Henneman, \& Long, 1960) have reported that if the $S$ is given the set of possible responses to be used during the experiment prior to the exposure of stimuli, the number of correct recognitions is increased. The data from this study suggest that certain sets may facilitate the recognition task more than other sets.

Studies by Solomon and Postman (1952) and Newbigging (1961) suggest a plausible explanation for the differences observed in this experiment. A stimulus which is presented for a brief duration may not be completely processed. Given that $\mathbf{S}$ processes some piece of the stimulus, his probability for making a correct response will be contingent on the utility of the cue. Whenever the ensemble of responses is known by the $S$ prior to exposure of a stimulus, the utility of the cue in recognition tasks is directly related to the discrimination redundancy of the expectancy set. If the stimuli have many common elements and few distinctive elements, and if only a random fragment of the stimulus is processed, the likelihood of a correct response is reduced. By minimizing the cue similarities, the high discrimination redundant set maximizes the usefulness of each cue in selecting the correct response.

\section{REFERENCES}

AVERBACH, E., \& CORIELL, A. S. Short-term memory in vision. Bell Sys. Tech. J., 1961, 40, 309-328.

EVANS, S. Redundancy as a variable in pattern perception. Psychol Bull. $1967,67,104-113$

FORGUS, R. Perception. New York: McGraw-Hill, 1966.

LONG, E. R., REID, L. S., \& HENNEMAN, R. H. An experimental analysis of set: variables influencing the identification of ambiguous visual stimulus objects. Amer. J. Psychol., 1960, 73, 553-562.

NEWBIGGING, P. L. The perceptual reintegration of frequent and infrequent words. Canad. J. Psychol., 1961, 15, 133-142.

REID, L. S., HENNEMAN, R. H., \& LONG, E. R. An experimental analysis of set: The effect of categorical restriction. Amer. J. Psychol, 1960, 73, 568-572.

SCHARF, B., ZAMANSKY, H. S., \& BRIGHTBILL, R. F. Word recognition with masking. Percept. \& Psychophys., 1966, 1, 110-112.

SCHILLER, P., \& WIENER, M. Monoptic and dichoptic visual masking. $J$. exp. Psychol., 1963, 66, 386-393.

SOLOMON, R. L., \& POSTMAN, L. Frequency of usage as a determinant of recognition thresholds for words. J. exp. Psychol, 1952, 43, 195-201.

SPERLING, G. The information available in brief visual presentations. Psychol Monogr., 1960, 74, No. 11.

(Accepted for publication March 29, 1968.) 